\title{
Rauchverzicht lohnt sich auch für Diabetiker
}

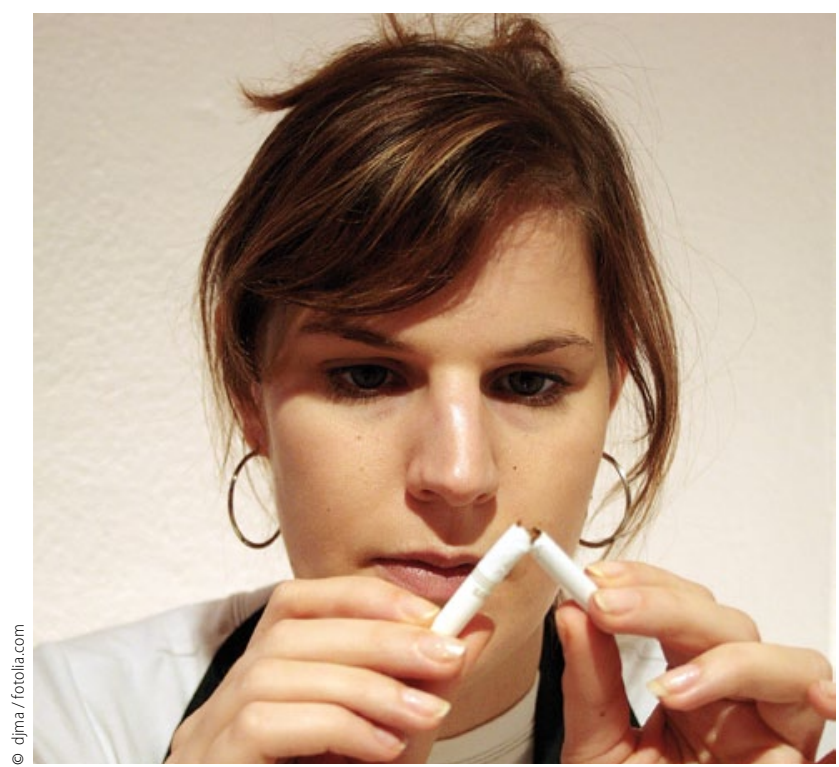

Den Glimmstängel sollten Diabetiker besser für immer ausmachen, denn nach Rauchverzicht sinkt auch ihr kardiovaskuläres Risiko.

Fragestellung: Verringern sich bei Diabetikern durch Aufgabe des Rauchens die kardiovaskulären Komplikationen ebenso deutlich wie bei Nichtdiabetikern?

Hintergrund: Rauchen ist ein bedeutsamer kardiovaskulärer Risikofaktor. Der Rauchverzicht hat aber häufig eine Gewichtszunahme zur Folge. Bei Diabetikern könnten dadurch die Stoffwechseleinstellung erschwert sowie diabetische mikro- und makrovaskuläre Spätkomplikationen begünstigt werden. Dann würden Diabetiker vom Rauchverzicht weniger profitieren als Nichtdiabetiker.

Patienten und Methodik: Die Daten stammen aus der Framingham-Studie und umfassen 3251 Probanden, die anfangs frei von kardiovaskulären Erkrankungen waren. Nachuntersuchungen erfolgten alle 4-6 Jahre. Nach im Mittel 25 Jahren waren 631 kardiovaskuläre Komplikationen (Todesfälle, Herzinfarkte, KHK, Schlaganfälle, pAVKs und Herzinsuffizienz) aufgetreten. In dieser Zeit ging der Anteil der Raucher von 31 auf $13 \%$ zurück.

Ergebnisse: Kurzzeit-Nichtraucher, d.h. Probanden, die weniger als vier Jahre das Rauchen

Clair C, Rigotti NA, Porneala B et al. Association of Smoking Cessation and Weight Change With Cardiovascular Disease Among Adults With and Without Diabetes. JAMA 2013;309(10):1014-21 aufgegeben hatten, nahmen mehr an Gewicht zu als Raucher, Langzeit-Nichtraucher (>4 Jahre) und Nichtraucher. Dies gilt gleichermaßen für Nichtdiabetiker und Diabetiker (Tab. 1). Langzeit-Nichtraucher hatten im Vergleich zu
T1 Gewichtsveränderungen und kardiovaskuläre Veränderungen nach Rauchverzicht

\begin{tabular}{lllll}
\hline Nicht-Diabetiker & Raucher & $\begin{array}{l}\text { Kurzzeit- } \\
\text { Nichtraucher }\end{array}$ & $\begin{array}{l}\text { Langzeit- } \\
\text { Nichtraucher }\end{array}$ & $\begin{array}{l}\text { Nicht- } \\
\text { Raucher }\end{array}$ \\
Gewicht kg/4 Jahre & $+1,2$ & $+3,0$ & $+0,9$ & $+1,2$ \\
CV Komplikationen & 1 & $-51 \%$ & $-54 \%$ & $-69 \%$ \\
Diabetiker & & & & \\
Gewicht/4 Jahre & \pm 0 & $+3,8$ & $+0,1$ & $+0,5$ \\
CV Komplikationen & 1 & $-51 \%$ & $-43 \%$ & $-51 \%$
\end{tabular}

Änderungen von Gewicht (kg/4 Jahre) und kardiovaskulären Komplikationen (prozentuale Änderung gegenüber Rauchern) bei Kurz- (<4 Jahre) und Langzeit- ( $>4$ Jahre) Abstinenzlern sowie Nichtrauchern.

Nichtrauchern und Rauchern keine signifikant unterschiedlichen Gewichtsänderungen.

Nichtraucher, aber auch Kurz- und Langzeit-Nichtraucher hatten - auch unter Berücksichtigung anderer Risikofaktoren sowie Alter und Geschlecht - weniger kardiovaskuläre Komplikationen als Raucher. Das gilt auch für Kurzzeit-Nichtraucher, die deutlich an Gewicht zunahmen. Es gab keine nennenswerten quantitativen Unterschiede zwischen Diabetikern und Nichtdiabetikern, obwohl statistische Signifikanz angesichts kleiner Fallzahlen bei den Diabetikern nicht erreicht wurde.

Schlussfolgerung: Ein Rauchverzicht führt bei Diabetikern und Nichtdiabetikern zu einem quantitativ ähnlichen Rückgang von kardiovaskulären Komplikationen.

\section{- Kommentar von Prof. Dr. med. Heinrich Holzgreve}

\section{Gewichtszunahme nur temporär}

Die Probanden dieser Studie bestätigen einmal mehr, dass nach der Aufgabe des Rauchens mit einer Gewichtszunahme zu rechnen ist. Innerhalb der ersten vier Jahre lag die Zunahme im Mittel bei 3-4kg. Nach mehr als vier Jahren Rauchverzicht sieht man aber keine Unterschiede mehr beim Vergleich mit Dauerrauchern und langfristig erfolgreichen Abstinenzlern. Rauchverzicht verhindert also kardiovaskuläre Folgekrankheiten trotz der - meist vorübergehenden - Gewichtszunahme. Diese wirkt sich bei Diabetikern nicht nachteilig aus, vielmehr profitieren sie ebenso wie Nichtdiabetiker.

Prof. Dr. med. Heinrich Holzgreve, München

\section{Korrektur zu Info Diabetologie 2-13 Seite 10}

Journalclubbeitrag „Metformin verlängert das Leben von Frauen

mit Ovarialkarzinom "von Prof. Heinrich Holzgreve, München.

Die Sulfonamide dienten nicht der Krebstherapie, wie irrtümlich von der Redaktion ergänzt, sondern wurden zuerst als antibakteriell wirksame Chemotherapeutika eingesetzt. Später entdeckte man ihre diuretischen und antidiabetischen Eigenschaften, was zur Entwicklung der

Thiaziddiuretika und der Sulfonylharnstoffe führte.

Die Redaktion bittet den Irrtum zu entschuldigen. 